\title{
Perfil dos pacientes traqueostomizados em uma Unidade de Terapia Intensiva adulto
}

\author{
Tracheostomized patients profile in an adult Intensive Care Unit \\ Perfil de pacientes traqueostomizados en una Unidade de Cuidados Intensivos para adultos
}

Recebido: 08/11/2021 | Revisado: 14/11/2021 | Aceito: 16/11/2021 | Publicado: 26/11/2021

Letícia Pedroso de Oliveira

ORCID: https://orcid.org/0000-0001-7409-5485

Centro Universitário da Amazônia, Brasil

E-mail: lelepedroso09@gmail.com

Marden Junio Sousa Ferreira

ORCID: https://orcid.org/0000-0002-3236-5237

Instituto Esperança de Ensino Superior, Brasil

E-mail: mardenjunio@gmail.com

Assis Junior Cardoso Pantoja

ORCID: https://orcid.org/0000-0002-8301-3135

Centro Universitário da Amazônia, Brasil

E-mail: assisfisio_jr@hotmail.com

Keren Thaís do Amaral Costa

ORCID: https://orcid.org/0000-0003-1200-5805

Centro Universitário da Amazônia, Brasil E-mail: kerenthais07@gmail.com

\begin{abstract}
Resumo
Este estudo refere-se à uma análise do perfil dos pacientes traqueostomizados em uma unidade de terapia intensiva adulto. Trata-se de um estudo descritivo, de característica documental, possuindo uma abordagem quantitativa e transversal, através da análise prontuários de pacientes hospitalizados no período de janeiro de 2020 a agosto de 2020 de ambos os gêneros, com idades entre 16 a 89 anos sendo aprovada pelo Comitê de Ética em Pesquisa com o número do parecer 4.898.929. Analisada estatisticamente através do software BioEstat. 5.3, utilizou-se $\mathrm{p} \leq 0,05$ para a significância estatística. Foi utilizado 51 prontuários no qual predominou o diagnóstico de COVID-19 com 84,3\% enquanto que $07,8 \%$ foram cirúrgico/oncológico, $03,9 \%$ infeccioso, $02,0 \%$ cirúrgico/cardiovascular e $02,0 \%$ por anemia falciforme. Em relação ao tempo total de ventilação mecânica (VM) quando comparados a alta e óbito tiveram uma associação significativa, observou-se que nos casos de óbito 76,5\% ficaram entre 16 a 30 dias na unidade de terapia intensiva e $23,5 \%$ ficaram acima de 30 dias. Com relação ao tempo de ventilação mecânica após confecção de traqueostomia não obteve associação significativa entre óbito e alta.
\end{abstract}

Palavras-chave: Desmame do ventilador; Traqueostomia; Fraqueza muscular.

\begin{abstract}
This study refers to an analyze of patients of an tracheostomized intensive care unit for adults. It's about a descriptive study with documental characteristic, containing a quantitave and transversal, approach through the analyze of patients medical record hospitalized during the period of January 2020 to august 2020 of both genders with the age between 16 and 89 years old, been approved by the committee of Ethics in Research with an opinion number of 4.898.929. Statistically analyzed through the Bioestat Software. 5.3 used $p \leq 0,05$ for the statistical significance. There were 51 medical records in which predominated the diagnosis of COVID-19 with 84,3\%, howbeit 07,8\% were surgical/oncological, $03,9 \%$ infectious, $02,0 \%$ surgical/cardiovascular and $02,0 \%$ cause by sickle cell anemia. Regarding the total time of mechanical ventilation (MV), when we compare the discharge and the deaths we had a significant result, it was observed in the deaths case that $76,5 \%$ were between 16 and 30 days in intensive care and $23,5 \%$ stayed more than 30 days. Regarding the time of the mechanical ventilation after making the tracheostomy there was no significant association between discharge and deaths.
\end{abstract}

Keywords: Ventilator weaning; Tracheostomy; Muscle weakness.

\section{Resumen}

Este estudio se refiere a un análisis del perfil de pacientes traqueostomizados en una unidad de cuidados intensivos para adultos. Se trata de un estudio descriptivo, con carácter documental, de abordaje cuantitativo y transversal, mediante el análisis de las historias clínicas de pacientes hospitalizados de enero de 2020 a agosto de 2020 de ambos sexos, con edades comprendidas entre 16 y 89 años, aprobadas por el Comité de Ética en Investigación con el dictamen número 4.898.929. Analizado estadísticamente mediante el software BioEstat. 5.3, p $\leq 0.05$ se utilizó para la significación estadística. Se utilizaron un total de 51 historias clínicas en las que predominó el diagnóstico de COVID-19 con 84,3\%, mientras que $07,8 \%$ fueron quirúrgicos / oncológicos, $03,9 \%$ infecciosos, $02,0 \%$ quirúrgicos / cardiovasculares y $02,0 \%$ por anemia falciforme. En relación al tiempo total de ventilación mecánica (VM) en comparación con el alta y la muerte tuvo una asociación significativa, se observó que em los casos de muerte $76,5 \%$ se encontraban entre 16 y 30 días em 
la unidad de cuidados intensivos y 23,5\% fueron mayores de 30 días. Con respecto a la duración de la ventilación mecánica después de la traqueotomía, no hubo asociación significativa entre muerte y alta.

Palabras clave: Destete del abanico; Traqueotomía; Debilidad Muscular.

\section{Introdução}

Os avanços na terapia intensiva permitiram que mais pacientes sobrevivessem às doenças críticas agudas, entretanto, estes avanços também criaram uma população de pacientes com dependência prolongada de Ventilação Mecânica (VM) e outras modalidades terapêuticas intensivas. Assim foi criada uma subdivisão de acordo com o tempo de suporte ventilatório, uma porcentagem menor se enquadra em pacientes de desmame curto (inferior à $48 \mathrm{~h}$ de ventilação) e uma minoria se encaixa no grupo de Ventilação Mecânica prolongada (VMP) (Loss et al., 2015).

A VMP é definida como um período igual ou superior a seis horas por dia sob VM ou também pelo tempo acima de 7 dias ininterrupto, contudo esse tempo é divergente na literatura pois alguns autores consideram acima de 21 dias sem desconexão (Loss et al., 2015). Sabe-se que o uso prolongado da ventilação mecânica pode desenvolver efeitos deletérios ou colaterais para o indivíduo, quanto maior o tempo de ventilação mecânica, maiores às chances de complicações relacionadas a ela (Loss et al., 2015).

Uma das principais indicações para a traqueostomia é a VMP pela possibilidade de redução do tempo de pressurização, redução do uso de sedativos e facilidade de mobilização. Atualmente, alguns estudos mostram que há benefícios significativos para uma traqueostomia precoce, esta utilizada em pacientes com tempo inferior a 7 dias de tubo orotraqueal (Sanabria et al., 2013).

A necessidade de um período de VM geralmente exige admissão a uma unidade de terapia intensiva (UTI), do mesmo modo, a VMP inclui o paciente no termo “crônico criticamente enfermo" (LOSS et al., 2015). No uso da VM prolongada haverá complicações patológicas podendo desenvolver efeitos adversos, esses incluem a via aérea, a parte pulmonar, hemodinâmica, neurológica, psicológicas e os cuidados com a pele (Gonzaga et al., 2007).

Portanto para impedir piores desfechos como os citados acima é iniciado um processo chamado desmame, este ocupa $40 \%$ do tempo total da ventilação, o sucesso desse processo depende de fatores clínicos e laboratoriais. Para se evitar danos para a via aérea superior e facilitar o desmame da VM é recomendado que a intubação seja convertida em traqueostomia de forma mais precoce, alguns autores reportam que após o décimo dia de intubação já deve ser discutido a confecção da traqueostomia, impactando de forma positiva nos desfechos (Ricz et al., 2011).

Sabendo da utilização da traqueostomia como forma de desmame em pacientes crônicos em uso de ventilação prolongada, ressaltamos que o objetivo deste estudo será analisar os perfil dos pacientes traqueostomizados na Unidade de Terapia Intensiva adulto.

\section{Metodologia}

Trata-se de uma pesquisa descritiva, de característica documental, possuindo uma abordagem quantitativa e transversal Costa e Barreto (2003), sendo desenvolvida na Unidade de terapia intensiva adulto. Constituída de indivíduos com idade entre 16 a 89 anos, de ambos os gêneros. A amostra foi composta através de análise de documentos, por meio de prontuários do período de janeiro de 2020 a agosto de 2020 .

Participaram desse estudo pacientes adultos com prontuários que possuiram dados com um tempo de ventilação mecânica mínimo de 7 dias de indivíduos que conseguiram receber alta e prontuários onde o desfecho foi óbito da unidade de terapia intensiva. Os dados coletados foram organizados, tabulados em planilha eletrônica por meio do software Microsoft Office Excel 2016. Os dados foram duplamente digitados, organizados e processados através da estatística descritiva em planilhas do programa Excel (Microsoft Office ${ }^{\circledR}$ 365). Foi realizada a análise estatística por meio da avaliação dos resultados através do software BioEstat 5.3. Em todos os testes utilizou-se $\mathrm{p} \leq 0,05$ para a significância estatística. 
A pesquisa seguiu a resolução $n^{\circ} 466 / 12$ CNS/CONEP que rege a pesquisa com seres humanos, CAAE: 49268521.6.0000.5193. Esta norma implica no anonimato dos indivíduos e no sigilo das informações adquiridas. Além disso, a pesquisa seguiu a Resolução de Julho de 2017, artigo $2^{\circ}$ onde explica o Termo de Compromisso de Uso de Dados (TCUD), afirmando que o estudo se compromete com o sigilo e a privacidade dos dados da pesquisa, assim como com o compromisso de que serão utilizados apenas para o estudo em questão.

Esta pesquisa faz parte de um estudo maior que foi intitulado: Perfil dos pacientes traqueostomizados em uma Unidade de Terapia Intensiva adulto sendo aprovado na dia 11 de agosto de 2021 pelo comite de etica e pesquisa do Centro de Ensino superior Piauiense LTDA com o número do parecer 4.898.929.

\section{Resultados}

Foram analisados 51 prontuários de pacientes que foram internados na unidade de terapia intensiva adulto. Notou-se que há uma pequena prevalência de gênero, onde o masculino representa 56,9\% ( $\mathrm{n}=29$ ) e o feminino 43,1\% (n=22), visto que cerca de 84,3\% $(n=43)$ desses pacientes foram internados na unidade de terapia intensiva com COVID-19, enquanto 07,8\% (n=4) foram por motivos cirúrgico/oncológico, 03,9\% $(\mathrm{n}=2)$ infeccioso, 02,0\% $(\mathrm{n}=1)$ cirúrgico/cardiovascular e $02,0 \%(\mathrm{n}=1)$ por anemia falciforme. (Tabela 1).

Tabela 1 - Dados sociodemográfico da amostra.

\begin{tabular}{|c|c|c|c|}
\hline \multirow{2}{*}{ Características da amostra } & \multicolumn{2}{|c|}{$\operatorname{Amostra}(n=51)$} & \multirow{2}{*}{ p-valor } \\
\hline & $\mathbf{F}$ & $\%$ & \\
\hline \multicolumn{4}{|l|}{ Sexo } \\
\hline Feminino & 22 & $43,1 \%$ & 0,4008 \\
\hline Masculino & 29 & $56,9 \%$ & \\
\hline \multicolumn{4}{|l|}{ Faixa-etária } \\
\hline De 16 a 29 anos & 05 & $09,8 \%$ & $0,0223 *$ \\
\hline De 30 a 39 anos & 06 & $11,8 \%$ & \\
\hline De 40 a 49 anos & 04 & $07,8 \%$ & \\
\hline De 50 a 59 anos & 11 & $21,6 \%$ & \\
\hline De 60 a 69 anos & 14 & $27,5 \%$ & \\
\hline De 70 a 79 anos & 09 & $17,6 \%$ & \\
\hline De 80 a 89 anos & 02 & $03,9 \%$ & \\
\hline \multicolumn{4}{|l|}{ COVID-19 } \\
\hline Sim & 43 & $84,3 \%$ & $<0,0001 *$ \\
\hline Não & 08 & $15,7 \%$ & \\
\hline \multicolumn{4}{|l|}{ Cirúrgico/Oncológico } \\
\hline Sim & 04 & $07,8 \%$ & $<0,0001 *$ \\
\hline Não & 47 & $92,2 \%$ & \\
\hline \multicolumn{4}{|l|}{ Infeccioso } \\
\hline Sim & 02 & $03,9 \%$ & $<0,0001$ \\
\hline Não & 49 & $96,1 \%$ & \\
\hline \multicolumn{4}{|l|}{ Cirúrgico/Cardiovascular } \\
\hline Sim & 01 & $02,0 \%$ & $<0,0001$ \\
\hline Não & 50 & $98,0 \%$ & \\
\hline \multicolumn{4}{|l|}{ Anemia Falciforme } \\
\hline Sim & 01 & $02,0 \%$ & $<0,0001$ \\
\hline Não & 50 & $98.0 \%$ & \\
\hline
\end{tabular}


$\mathrm{Na}$ análise de associação entre o diagnóstico de COVID-19 e as outras patologias observou-se que não houve correlação significativa visto que $84,3 \%$ ( $n=43$ ) da amostra foi composta por pacientes acometidos por COVID-19, ressaltando o período em que foi coletado os dados (Tabela 2).

Tabela 2 - Associação entre o diagnóstico de COVID-19 e outras patologias, N=51. Santarém-PA, 2021.

\begin{tabular}{ccc}
\hline Diagnósticos & \multicolumn{2}{c}{} \\
\cline { 2 - 3 } & $\mathbf{F}$ & $\%$ \\
COVID-19 & 04 & $84,3 \%$ \\
Cirúrgico/Oncológico & 02 & $07,8 \$$ \\
Infeccioso & & $03,9 \%$ \\
Cirúrgico/Cardiovascular & 01 & $02,0 \%$ \\
Anemia Falciforme & 01 & $02,0 \%$ \\
\hline
\end{tabular}

Fonte: Autores.

Referente ao motivo que levou a necessidade de ventilação mecânica $(86,3 \%)$ era de insuficiência respiratória aguda tipo III, $(14,7 \%)$ era de insuficiência respiratória tipo I e apenas $(05,9 \%)$ era relacionados a outros distúrbios que levaram a necessidade de via aérea artifical (Tabela 3). Quando observados os valores de óbito e alta, os pacientes que evoluíram com óbito 100,0\% (n=17) estavam no grupo que foram intubados com insuficiência respiratória aguda tipo III. (Tabela 3). Em relação ao tempo total de ventilação mecânica quando comparados a alta e óbito tiveram uma associação significativa, observou-se que nos casos de óbito 76,5\% (n=13) ficaram entre 16 a 30 dias na unidade de terapia intensiva e 23,5\% (n=4) ficaram acima de 30 dias e já os pacientes que tiveram alta 47,1\% (n=16) ficaram entre 16 a 30 dias. Com relação ao tempo de ventilação mecânica após confecção de traqueostomia não obteve associação significativa entre óbito e alta. (Tabela 3). 
Tabela 3 - Resultados da associação entre as características clínicas relacionadas a VM e o desfecho clínico, N=51. SantarémPA, 2021.

\begin{tabular}{|c|c|c|c|c|c|c|c|}
\hline \multirow{3}{*}{ Características clínicas relacionadas a VM } & \multicolumn{4}{|c|}{ Desfecho } & \multirow{2}{*}{\multicolumn{2}{|c|}{ Geral $(n=51)$}} & \multirow{3}{*}{ p-valor } \\
\hline & \multicolumn{2}{|c|}{ Óbito $(n=17)$} & \multicolumn{2}{|c|}{ Alta $(n=34)$} & & & \\
\hline & $\mathbf{f}$ & $\%$ & $\mathbf{f}$ & $\%$ & $\mathbf{f}$ & $\%$ & \\
\hline \multicolumn{8}{|l|}{ Motivo ou necessidade de VM } \\
\hline Cirurgia & 00 & $0,0 \%$ & 02 & $05,9 \%$ & 02 & $03,9 \%$ & 0,0757 \\
\hline IRPA- I & 00 & $0,0 \%$ & 05 & $14,7 \%$ & 05 & $09,8 \%$ & \\
\hline IRPA- III & 17 & $100,0 \%$ & 27 & $79,4 \%$ & 44 & $86,3 \%$ & \\
\hline \multicolumn{8}{|l|}{ Tempo total de VM (dias) } \\
\hline Até 15 dias & 00 & $0,0 \%$ & 05 & $14,7 \%$ & 05 & $09,8 \%$ & $0,0497 *$ \\
\hline De 16 a 30 dias & 13 & $76,5 \%$ & 16 & $47,1 \%$ & 29 & $56,9 \%$ & \\
\hline Acima de 30 dias & 04 & $23,5 \%$ & 13 & $38,2 \%$ & 17 & $33,3 \%$ & \\
\hline \multicolumn{8}{|l|}{ Tempo em dias de VM pós TQT } \\
\hline Até 07 dias & 07 & $41,2 \%$ & 12 & $35,3 \%$ & 19 & $37,3 \%$ & 0,8958 \\
\hline De 08 a 15 dias & 07 & $41,2 \%$ & 13 & $38,2 \%$ & 20 & $39,2 \%$ & \\
\hline De 16 a 30 dias & 02 & $11,8 \%$ & 07 & $20,6 \%$ & 09 & $17,6 \%$ & \\
\hline Acima de 30 dias & 01 & $5,9 \%$ & 02 & $05,9 \%$ & 03 & $05,9 \%$ & \\
\hline \multicolumn{8}{|l|}{ Volume acentuado de secreções } \\
\hline Sim & 16 & $94,1 \%$ & 31 & $91,2 \%$ & 47 & $92,2 \%$ & 0,8552 \\
\hline Não & 01 & $5,9 \%$ & 03 & $08,8 \%$ & 04 & $07,8 \%$ & \\
\hline \multicolumn{8}{|l|}{ Falha de extubação } \\
\hline Sim & 00 & $0,0 \%$ & 07 & $20,6 \%$ & 07 & $13,7 \%$ & 0,0781 \\
\hline Não & 17 & $100,0 \%$ & 27 & $79,4 \%$ & 44 & $86,3 \%$ & \\
\hline \multicolumn{8}{|l|}{ Novas infecções após a TQT } \\
\hline Sim & 16 & $94,1 \%$ & 27 & $79,4 \%$ & 43 & $84,3 \%$ & 0,3207 \\
\hline Não & 01 & $5,9 \%$ & 07 & $20,6 \%$ & 08 & $15,7 \%$ & \\
\hline \multicolumn{8}{|l|}{ PCR } \\
\hline Sim & 03 & $17,6 \%$ & 02 & $05,9 \%$ & 05 & $09,8 \%$ & 0,4174 \\
\hline Não & 14 & $82,4 \%$ & 32 & $94,1 \%$ & 46 & $90,2 \%$ & \\
\hline
\end{tabular}

Fonte: Autores.

De acordo com a análise, quando comparado a média de idade, tempo de VM em dias e tempo de ventilação após confecção da traqueostomia entre os casos de óbito e alta não observou-se uma caracterização importante no tempo de permanência na UTI após o desmame da VM e o tempo para decanulação após confecção da TQT na amostra que evoluiu para óbito ou que evoluiu para alta. (Tabela 4). 
Tabela 4 - Resultado da comparação entre a idade, tempo na ventilação mecânica, e tempo na ventilação mecânica após traqueostomia entre os casos de óbito e alta, N=51. Santarém-PA, 2021.

\begin{tabular}{|c|c|c|c|c|}
\hline & Óbito & Alta & Geral & \\
\hline Variáveis & $\begin{array}{c}\text { Média } \pm \\
\text { Desvio-padrão }\end{array}$ & $\begin{array}{c}\text { Média } \pm \\
\text { Desvio-padrão }\end{array}$ & $\begin{array}{c}\text { Média } \pm \\
\text { Desvio-padrão }\end{array}$ & p-valor* \\
\hline Idade (anos) & $58,9 \pm 15,0$ & $54,1 \pm 18,5$ & $55,7 \pm 17,4$ & 0,3535 \\
\hline Tempo de VM (dias) & $28,9 \pm 15,2$ & $30,9 \pm 18,8$ & $30,2 \pm 17,6$ & 0,7015 \\
\hline Tempo de VM pós TQT (dias) & $13,0 \pm 13,3$ & $13,8 \pm 16,7$ & $13,5 \pm 15,5$ & 0,8704 \\
\hline $\begin{array}{l}\text { Tempo de permanência na UTI após desmame da } \\
\qquad \text { VM (dias) }\end{array}$ & - & $9,0 \pm 6,5$ & - & - \\
\hline $\begin{array}{l}\text { Tempo para decanulação após confecção da TQT } \\
\text { (dias) }\end{array}$ & - & $8,6 \pm 16,7$ & - & - \\
\hline
\end{tabular}

Fonte: Autores.

A correlação entre idade e tempo de ventilação foram denominados 0 para óbito e 1 para alta, notou-se que não houve significância na correlação dessas variáveis ou seja os desfechos 0 e 1 não tiveram relação para as variáveis de idade, tempo de ventilação e tempo de ventilação após confecção da TQT (Tabela 5).

Tabela 5 - Resultado da correlação entre a idade, tempo de VM e tempo de VM pós TQT com o tipo de desfecho clinico, $\mathrm{N}=51$. Santarém-PA, 2021.

\begin{tabular}{lcc}
\hline \multirow{2}{*}{ Variáveis } & \multicolumn{2}{c}{ Desfecho (0-óbito e 1-alta) } \\
\cline { 2 - 3 } & r & p-valor* \\
\hline Idade (anos) & $-0,133$ & 0,3535 \\
Tempo de VM (dias) & 0,06 & 0,7015 \\
Tempo de VM pós TQT (dias) & 0,02 & 0,8704 \\
\hline
\end{tabular}

Fonte: Autores.

Relacionado aos resultados do maior grau de funcionalidade no dia da alta da terapia intensiva, nota-se, que a 27,5\% dos pacientes pós desmame da VM apresentou limitação nas suas funcionalidades, apresentando restrição ao leito. Porém a maioria apresentou certo grau de funcionalidade como por exemplo sentado beira leito de forma ativa ou passiva $29,5 \%$. As demais funcionalidades apresentaram porcentagem baixa mas que se destacam pela independência funcional, como, sentar fora do leito, somente $5,8 \%$, ficar em pé $2,9 \%$ e tiveram a capacidade de andar apenas $5,8 \%$ desses pacientes. (Tabela 6). 
Tabela 6 - Resultado do grau de funcionalidade após desmame de VM, N=34. Santarém-PA, 2021.

\begin{tabular}{ccc} 
Maior Grau de Funcionalidade & F & \% \\
\hline $\begin{array}{c}\text { Acamado ou mudança de decúbito- Ativa, assistida e } \\
\text { Passiva }\end{array}$ & 14 & $27,5 \%$ \\
Sedestação beira leito- Ativa ou assistida & 15 & $29,5 \%$ \\
Sedestação fora do leito & 02 & $2,9 \%$ \\
Ortostase & 01 & $5,8 \%$ \\
Deambula & 02 & $5,8 \%$
\end{tabular}

Fonte: Autores.

Referente ao resultado de força muscular periférica no dia da alta, nota-se que $80,4 \%(n=25)$ tiveram força de pressão palmar através da dinamometria de 01 a $10 \mathrm{~kg} / \mathrm{F}$ o que significa uma perda funcional significante onde apenas 6 pacientes apresentaram forças que permitem funcionalidade. (Tabela 7).

Tabela 7: Resultado de força muscular no dia da alta, N=31. Santarém PA.

\begin{tabular}{ccc} 
Informe dinamometria $(\mathbf{K g} / \mathbf{F})$ & $\mathbf{F}$ & $\mathbf{\%}$ \\
\hline De 01 a 10 & 25 & $80,4 \%$ \\
De 11 a 20 & 04 & $12,9 \%$ \\
De 21 a 35 & 02 & $6,4 \%$ \\
\hline
\end{tabular}

Fonte: Autores.

\section{Discussão}

Para traçar o perfil dos pacientes submetidos a traqueostomia durante a internação na unidade de terapia intensiva adulto, os resultados apontaram que o gênero masculino (72,2\%) predominou sobre o feminino (27,7\%) (Pasini et al., 2004). Resultados semelhantes foram encontrados no estudo feito por (De Souza et al., 2021) onde a taxa de internação foi maior para o gênero masculino e para pacientes com idade mais avançada de ambos os gêneros. Dados que corroboram com o presente estudo onde apresenta uma pequena prevalência do gênero masculino $(56,9 \%)$ sobre o gênero feminino $(43,1 \%)$ com idade entre 16 a 89 anos. Segundo (Souza, Hojo, Batista, silva \& Guidoni, 2021), devido a pandemia da COVID-19 a prevalência ainda sim era do gênero masculino, entretanto com a média de idade para internações acima de 65 anos.

Quando associado diagnóstico de COVID-19 e as outras patologias, observou-se que a maioria dos pacientes em estudo $(84,3 \%)$ foram internados na unidade de terapia intensiva com diagnóstico clínico de Covid-19, tendo em vista que o período de coleta foi feito durante a pandemia de COVID-19, o que justifica a ocupação da maior parte da amostra sendo causa de internações. Tais dados corrobram ao estudo de (Teich et al., 2020) onde a maioria dos pacientes foram internados em UTI devido à insuficiência respiratória hipoxêmica aguda ocasionada pela Covid-19, que necessitava de suporte ventilatório. Da mesma forma às informações do estudo de (Jardim et al., 2021) onde os dados apontam o aumento da quantidade de pacientes 
que necessitaram de internação hospitalar, especialmente daqueles que necessitaram de internação na UTI. Ao se comparar os diagnósticos mais comuns, houve uma diminuição nos casos e aumento da demanda de atendimento para COVID-19.

Consequentemente, no presente estudo não houve correlação entre os diagnósticos de COVID-19 entre as outras variáveis patológicas. No entanto, na literatura observa-se uma associação significativa, visto que a junção da pandemia de COVID-19 e doenças crônicas ficou reforçado ainda mais a importância do controle dessas patologias e entender como elas se desenvolvem, é essencial para conseguirmos diminuir o número de novas notificações, reinfecções e óbitos, (Souza, 2021). Nessa perspectiva, um estudo realizado por (Guan et al., 2020) constatou que os indivíduos com multicomorbidades tiveram riscos significativamente mais altos de alcançar um desfecho negativo como ventilação mecânica invasiva ou óbito, em comparação com aqueles que tinham uma única comorbidade, o risco foi ainda maior, frente àqueles sem comorbidades.

Analisando a associação entre as características clínicas relacionadas a ventilação mecânica e o desfecho clínico da amostra, no geral, observa-se que todos os casos que evoluíram a óbito apontaram insuficiência respiratória aguda tipo III (100\%). Já os dados dos pacientes que tiveram alta hospitalar, mostram que a maioria deles $(79,4 \%)$ apresentou também insuficiência respiratória aguda tipo III, além de outros casos cirúrgicos e de insuficiência respiratória aguda tipo I. Assim como no estudo de (Gomes, 2021) onde o principal motivo da admissão na UTI foi a insuficiência respiratória aguda e as principais indicações de traqueostomia foram o desmame difícil e/ou prolongado e ventilação mecânica prolongada. Corroborando com os resultados de (Gomes et al., 2021) que apontam a insuficiência respiratória tipo III como o principal motivo que levou a necessidade de ventilação mecânica.

Referente ao tempo total de ventilação mecânica verificou-se que nos casos de óbito, a maioria deles $(76,5 \%)$ foi submetida a ventilação mecânica por um tempo de 16 a 30 dias, igualmente nos pacientes que tiveram alta hospitalar, onde a maioria deles $(47,1 \%)$ também foi submetida a ventilação mecânica entre 16 e 30 dias. No tempo em dias de ventilação mecânica após a confecção de traqueostomia, a maioria dos casos de óbito passaram até 15 dias em ventilação mecânica, onde variáveis de "até sete dias" e "de 08 a 15 dias", apresentaram o mesmo resultado (41,2\%). Já os casos de alta, a maioria permaneceu de 08 a 15 dias em ventilação mecânica (38,2\%). Esses dados apontam que o tempo de ventilação não interferiu no desfecho clinico, corroborando com (Guan et al., 2020) que afirmam que os desfechos negativos estão associados ao estado de saúde de cada paciente, onde as comorbidades adjacentes influenciarão na alta ou óbito dos casos clínicos. Assim como (Mota et al., 2021) que afirmam que o desfecho clínico de cada paciente depende das suas condições fisiológicas e da gravidade da doença.

Percebe-se então que basicamente, os casos de óbito e alta tiveram a mesma média de tempo de ventilação mecânica, indicando que o fator de desfecho não é a traqueostomia e sim o estado da doença crítica de cada paciente, como a condição pulmonar, infecciosa, o estado nutricional, muscular, fatores que indicam o tempo de permanência do paciente na ventilação mecânica e na UTI. Em contrapartida (Aranha, 2007) e (Mota et al., 2021), apontam que, quanto maior o tempo de ventilação mecânica, maior o índice de mortalidade. E para (Mota et al. 2021) esses resultados podem ser atribuídos ao fato de que os pacientes traqueostomizados foram submetidos a um período maior de ventilação mecânica, como também ao fato de que muitos pacientes submetidos à ventilação mecânica tiveram maior taxa de mortalidade, provavelmente por serem mais graves.

Ao analisar o componente insucesso na extubação, percebe-se que tanto os casos de óbito (100\%) quanto os casos de alta $(79,4 \%)$ não apresentaram falhas. Diferente do estudo de (Medeiros et al., 2019) onde os autores mostram falhas na extubação, na qual os fatores que levaram ao insucesso do processo foram por dificuldade de expectoração ou aumento da secreção, ausência de deglutição, presença de disfagia, presença de estenose traqueal e infecção pulmonar, favorecendo o falhas no teste de respiração espontânea. Em nosso estudo, a demanda da maioria dos casos era de síndrome de desconforto respiratório agudo, onde os pacientes, por vezes, ainda estariam recebendo sedação e bloqueio neuromuscular, impossibilitando o teste de respiração espontânea a extubação. Por este fato, esses pacientes, possivelmente, receberam a traqueostomia sem falhas na extubação. Tais dados corroboram com os estudos (Bordon et al., 2021) onde indivíduos com síndrome de desconforto 
respiratório agudo na categorização moderado e grave evoluíram em grande parte para traqueostomia e desmame gradual da ventilação mecânica sem falhas.

Relacionado a citação anterior, quando investigado o desmame da ventilação mecânica em indivíduos com síndrome de desconforto respiratório agudo, em consequência da COVID-19, a literatura apresenta dados que apontam que mais de $40 \%$ dos pacientes necessitaram de mais um teste de respiração espontânea para alcançar a extubação, ou atingiram a saída da ventilação mecânica após 7 dias (Bordon et al., 2021; Nash et al., 2020). Já (Lages \& Neumann, 2011) observaram que os pacientes com maior tempo de traqueostomia apresentaram maior taxa de insucesso, onde as causas estão relacionadas principalmente à retenção de secreção.

Referente aos casos de óbito, a idade média foi de 58,9 e de alta foi de 54,1. Já o tempo de permanência na UTI após desmame da ventilação mecânica em dias, a média foi de 9,0. A correlação entre idade, tempo de ventilação mecânica e o tempo de ventilação mecânica pós traqueostomia nos casos de óbito e alta mostrou que não houve relação entre o desfecho clínico e as variáveis em questão. A idade não parece ser um preditor importante, segundo este estudo, pois o tempo de ventilação independe da idade do paciente, mas sim do estado da doença crítica. No entanto, os dados se opõem aos estudos de (Araujo \& Soares, 2020), que mostram uma forte correlação inversamente proporcional para a idade e o tempo de internação, ou seja, quanto menor a idade mais provável que possua um menor tempo de internação, por outro lado, houve uma correlação positiva entre a mortalidade e o avançar da idade.

Na relação do tempo de internação com a idade, foi observado maior tempo de internação para pacientes mais velhos. (Araujo \& Soares, 2020). Fato que é condizente com a literatura, a qual afirma que a letalidade da doença é diretamente proporcional a idade do paciente, especialmente acima dos 80 anos, e as suas comorbidades prévias, principalmente doenças respiratórias, onde a mortalidade decorre de um quadro clínico com insuficiência respiratória e/ou choque séptico e/ou falência de múltiplos órgãos. (Gallasch, 2020; Wang et al., 2020; Chen et al., 2020). Em oposição, o estudo prévio de (Frutos-Vivar et al., 2005) relatou que pacientes com traqueostomia tiveram maior tempo de internação, tanto na UTI quanto no hospital, quando comparados com pacientes sem traqueostomia. Outro estudo relatou que a traqueostomia não interfere no tempo de internação hospitalar (Barquist et al., 2006).

Ao analisar, o tempo para decanulação após confecção da traqueostomia teve a média de 8,6, nos casos de alta. Não houve diferença significativa entre a amostra dos casos de óbito e a amostra dos casos de alta. Igualmente no estudo de (Melgaço et al., 2021) no qual a média de tempo de decanulação foi de oito dias e de liberação de via oral de 9,5 dias. Já a análise de (Archer, Iezzi, \& Gilpin, 2021) em pacientes com COVID traqueostomizados, a maioria (70,9\%) foram decanulados sem falhas, havendo o tempo médio de utilização de traqueostomia de 19 dias. Para (Medeiros et al., 2019), os fatores apontados como indicativos de sucesso no processo de decanulação são estabilidade clínica e hemodinâmica, nível de consciência alerta e paciente colaborativo, não necessidade de ventilação mecânica, não dependência de umidificação, bom gerenciamento das secreções e ausência de broncoaspiração. Dentre as etapas mais importantes no processo de decanulação, destacam-se desinsuflação do Cuff; permeabilidade de vias aéreas, avaliação da deglutição, gerenciamento das secreções e treino de oclusão da traqueostomia.

Segundo (Souza et al., 2019) o acúmulo de agressões que o indivíduo adquire durante sua internação na unidade de terapia intensiva são adversas o que inclui a ventilação mecânica invasiva, uso de sedativos, bloqueadores neuromusculares e sepses. Ou seja quanto mais grave está o paciente maior será a evolução do déficit físico e funcional do internado. Em nosso estudo, observou-se que 80,4\% dos pacientes apresentaram força de pressão palmar através da dinamometria de 01 a $10 \mathrm{~kg} / \mathrm{F}$. A maioria dos pacientes se encontrava com uma força de preensão palmar inferior a $11 \mathrm{~kg} / \mathrm{F}$ (34\%), indicando fraqueza muscular adquirida na UTI. Consequentemente observa-se essa amostra tem alta incidência para desenvolver a síndrome pós terapia intensiva (SPTI). Para (Robinson et al., 2018) a SPTI caracteriza-se por alterações físicas, cognitivas e psiquiátricas, que têm o 
potencial de levar à redução da qualidade de vida dos pacientes, no qual mostrou que apenas após 3 meses da alta hospitalar os pacientes conseguiram alcançar sua independência funcional.

Em afirmação a citação acima, valores encontrados no estudo de (Aarden et al., 2019), no qual em homens inferiores a $11 \mathrm{~kg} / \mathrm{F}$ e inferiores a $7 \mathrm{Kg} / \mathrm{F}$ em mulheres sugerem o diagnóstico funcional de um paciente enfraquecido. De acordo com (Santos et al., 2017) indica que casos de desmame da ventilação mecânica que alcançam $23 \mathrm{Kg} / \mathrm{F}$ na avaliação da força muscular periférica com dinamômetro de preensão palmar estão com ganho para o controle de tronco, proteção de suas vias aéreas e, na maioria dos casos, atingir a deambulação. Quando estabelecido o resultado de força muscular no dia da alta não foi observada correlação positiva, haja vista que, uma grande parcela da amostra analisada se encontrava em desmame difícil e prolongado, e apenas isso já é suficiente para reduzir a força de preensão palmar, independentemente da idade do indivíduo (Chlan, 2015).

Analisando ainda o mesmo tema, de acordo com (Roque, Souza \& Tavares, 2017) estima-se que 30 a 60\% desses pacientes internados a um período prolongado desenvolvem a fraqueza muscular adquirida na UTI. Os dados relacionados ao resultado do grau de funcionalidade após desmame da ventilação mecânica indicam que a maioria dos pacientes $(27,5 \%)$ apresentaram maior grau de funcionalidade acamado ou na mudança de decúbito ativa, assistida e passiva, seguida de sedestação beira leito ativa ou assistida (29,5\%), observa-se que não houve tanta significância estatística, visto que $(29,5 \%)$ dos pacientes tiveram capacidade de controle de tronco com sedestação beira leito. A partir disso nota-se que grande parte desses pacientes tem maiores chances de adquirir a SPTI. De acordo com (Maia et al., 2020), devido ao longo período de internação os pacientes estão mais propensos a alterações, apresentando um alto nível de descondicionamento e desfechos não favoráveis a curto e longo prazo. Quando estabelecido um teste de caminhada de 6 minutos notou-se que a maioria dos pacientes apresentaram comprometimento funcional, onde, (49\%) dos pacientes conseguiram chegar na marca da faixa esperada, entretanto (75\%) chegaram a marca esperada somente um ano após a alta hospitalar.

\section{Conclusão}

Conforme os resultados obtidos através dessa pesquisa e atendendo o objetivo de traçar o perfil dos pacientes traqueostomizados conclui-se com esta que nossa amostra apresentou uma prevalência de casos de COVID 19 o que deixa claro os tempos de pandemia e sua influência nas intervenções com traqueostomia. Outros motivos menos significantes estatisticamente foram cirúrgico/oncológico, infeccioso, cirúrgico/cardiovascular e anemia falciforme.

Notou-se que dos pacientes com traqueostomia que vieram a óbito todos tiveram como motivo principal a insuficiência respiratória aguda tipo III, entretanto, a IRPA está presente na maioria dos pacientes que tiveram sucesso e evoluindo com alta da UTI.

Conclui-se ainda que o tempo e a idade não foram motivos significantes para os casos de óbitos e alta, observou que há uma caracterização importante no tempo de permanência na UTI após o desmame da VM e o tempo para descanulação após confecção da TQT.

Portanto, essa pesquisa mostrou que a temática tem uma escassez de pesquisas disponíveis na literatura científica com boas evidências, mostrando a necessidade de mais estudos sobre o presente tema e maiores investimentos em pesquisas, visto ser um assunto de suma importância, principalmente em período de pandemia e correlacionando a um procedimento de muita frequência em ambientes de terapia intensiva, tornando algo sugestivo para futuros trabalhos ou artigos, levando em consideração o diagnóstico, tempo de VM, idade, motivos/necessidades.

\section{Referências}

Aarden J. J., Schaaf M. V., Esch M. V., Reichardt L. A., Seben R. V., Bosch J. A., Twisk J. W. R., Buurman B. M., Engelbert R. H. H. (2019). Muscle strength is longitudinally associated with mobility among older adults after acute hospitalization: The Hospital-ADL study. PloS ONE, v. 14, n. 7, p. 1-11. 
Aranha S. C., Mataloun, S. E., Moock, M., \& Ribeiro, R. (2007). Estudo comparativo entre traqueostomia precoce e tardia em pacientes sob ventilação mecânica. Revista brasileira de terapia intensiva, 19, 444-449.

Araujo, C. N. F., Soares, O. A. B. (2020). Perfil dos pacientes baixados por Covid-19 no Hospital Geral de Belém.

Archer, S. K., Iezzi, C. M., \& Gilpin, L. (2021). Swallowing and Voice Outcomes in Patients Hospitalized With COVID-19: An Observational Cohort Study. Archives of Physical Medicine and Rehabilitation, 102(6), 1084-1090.

Barquist, E. S., Amortegui, J., Hallal, A., Giannotti, G., Whinney, R., Alzamel, H., \& MacLeod, J. (2006). Tracheostomy in ventilator dependent trauma patients: a prospective, randomized intention-to-treat study. Journal of Trauma and Acute Care Surgery, 60(1), 91-97.

Bordon, J., Akca, O., Furmanek, S., Cavallari, R. S., Suliman, S., Aboelnasr, A, Sinanova, B., \& Ramirez, J.A. (2021). Acute Respiratory Distress Syndrome and Time to Weaning Off the Invasive Mechanical Ventilator among Patients with COVID-19 Pneumonia. J. Clin. Med., 10(2935):1-11.

Costa M.F. L; Barreto S.M. (2003). Types of epidemiologic studies: basic concepts and uses in the area of aging. Epidemiologia e Serviço de Saúde. v, 12, n. 4, p. 189-201.

Chen, N., Zhou M., Dong X., Qu J., Gong F., Han Y., Qiu Y., Wang J., Liu Y., Wei Y., Xia J., Yu T., Zhang X., Zhang L. (2020). Epidemiological and clinical characteristics of 99 cases of novel coronavirus pneumonia in Wuhan, China: a descriptive study. The lancet, 395(10223), 507-513.

Chlan, L. L. (2015). Description of Peripheral Muscle Strength Measurement and Correlates of Muscle Weakness in Patients Receiving Prolonged Mechanical Ventilatory Support. Am J Crit Care, 24(6):91-98.

De Souza, F. S. H., Hojo-Souza, N. S., Batista, B. D. D. O., da Silva, C. M., \& Guidoni, D. L. (2021). On the analysis of mortality risk factors for hospitalized COVID-19 patients: A data-driven study using the major Brazilian database. PloS one, 16(3), e0248580

Frutos-Vivar, F., Esteban, A., Apezteguía, C., Anzueto, A., Nightingale, P., González, M., ... \& International Mechanical Ventilation Study Group. (2005). Outcome of mechanically ventilated patients who require a tracheostomy. Critical care medicine, 33(2), 290-298.

Gallasch, C. H., da Cunha, M. L., de Souza Pereira, L. A., \& Silva-Junior, J. S. (2020). Prevenção relacionada à exposição ocupacional do profissional de saúde no cenário de COVID-19. Revista Enfermagem UERJ, 28, 49596.

Gomes, G. P. D. M. (2021). Traqueostomia em unidade de terapia intensiva: características demográficas, clínicas e principais desfechos.

Gomes, G. S., Leite, M. M., da Silva, M. C. B., Ferreira, M. J. S., \& dos Santos, L. P. C. (2021). Avaliação da funcionalidade e força muscular periférica pós desmame da configuração mecânica em uma UTI adulto. Research, Society and Development, 10 (13), e554101321477-e554101321477.

Gonzaga, A. D., Figueira, B. B. D., Sousa, J. M. A., \& Carvalho, W. B. D. (2007). Tempo de ventilação mecânica e desenvolvimento de displasia broncopulmonar. Revista da Associação Médica Brasileira, 53, 64-67.

Guan, W. J., Liang, W. H., Zhao, Y., Liang, H. R., Chen, Z. S., Li, Y. M., ... \& He, J. X. (2020). Comorbidity and its impact on 1590 patients with COVID-19 in China: a nationwide analysis. European Respiratory Journal, 55(5).

Jardim, T. V., Jardim, F. V., Jardim, L. M. V., Coragem, J. T., Castro, C. F., Firmino, G. M., \& Jardim, P. C. B. V. (2021). Alterações no Perfil dos Pacientes atendidos no Pronto Socorro durante o Surto de COVID-19 em um Hospital Geral Especializado em Tratamento Cardiovascular no Brasil. Arquivos Brasileiros de Cardiologia, 116, 140-143.

Lages, N. C. L., \& Neumamm, L. B. A. (2011). Decanulação em traqueostomia: uma abordagem prática [acesso em 18de julho de 2013 ]. Revista InterFISIO.

Loss, S. H., Oliveira, R. P. D., Maccari, J. G., Savi, A., Boniatti, M. M., Hetzel, M. P. \& Teixeira, C. (2015). A realidade dos pacientes que necessitam de ventilação mecânica prolongada: um estudo multicêntrico. Revista Brasileira de Terapia Intensiva, 27, 26-35.

Maia, H. F; PINTO, E. B.; Aleluia, Í. R. S.; Cavalcante, L. L. R.; Pedreira, R. B. S.; Silva, T. de J.; Souza, T. S. de; Pinto, J. M.; Pinto Junior, E. P. (2021). Fisioterapia e COVID-19: das repercussões sistêmicas aos desafios para oferta de reabilitação. Construção de conhecimento no curso da pandemia de COVID19: aspectos biomédicos, clínico-assistenciais, epidemiológicos e sociais. Salvador: Edufba, v. 1.

Medeiros, A. I. C., Silva, L. S. \& Bastos, V. P. D. (2019). Perfil clínico e índices preditativos de desmame de pacientes extubados em uma unidade de terapia intensiva em fortaleza, CE. ASSOBRAFIR ciência, 6 (3), 33-42.

Melgaço, S. A., Vicente L. C. C., Gama A. C. C. (2020). Analysis of decannulation time and oral intake recovery in oral cancer patients. Belo Horizonte (MG), Brasil.33(4).

Mota, J. D. H., Rodrigues, Y. D. S., \& Souza, F. D. S. L. D. (2021). Análise do tempo de retirada do respirador artificial no paciente submetido a traqueostomia precoce e após sete dias de ventilação mecânica invasiva. Fisioterapia e Pesquisa, 27, 306-311.

Nash D. S., Khoundabi B., Azar G. M., Maleakmohammad, M., Jamaati, H., \& Hashemian, S. M. (2020). Beneficial Outcomes of Early Tracheostomy in Patients Requiring Prolonged Mechanical Ventilation. Tanaffos, 19(4): 350-355.

Pasini, R. L., Roquejani, A. C., de Oliveira, R. A. R. A., Soares, S. M. P., \& Araújo, S. (2004). Perfil das traqueostomias na Unidade de Terapia Intensiva. Neurologia, 2(11), 1 .

Ricz, H. M. A., de Mello Filho, F. V., de Freitas, L. C. C., \& Mamede, R. C. C. M. (2011). Traqueostomia. Medicina (Ribeirão Preto), $44(1)$, 63-69.

Robinson C. C., Rosa R. G., Kochhann R., Schneider D., Sganzerla D., Dietrich C., Sanchez E. C., Dutra F. H., Oliveira M. Q., Anzolin L. B., Menezes S. F.,

Jeffman R., Souza D., Silva S. F., Cruz L. N., Boldo R., Cardoso J. R., Birriel D. C., Gamboa M. N., Machado A. S., Andrade J. M .S., Alencar C., Teixeira M. C., Vieira S. R. R., Moreira F. C., Amaral A., Silveira A. P. M., Teles J. M. M., Oliveira D. C., Oliveira L. C. J., Castro L. C., Silva M. S., Neves R. T., Gomes 
Research, Society and Development, v. 10, n. 15, e280101522996, 2021 (CC BY 4.0) | ISSN 2525-3409 | DOI: http://dx.doi.org/10.33448/rsd-v10i15.22996

R. A., Ribeiro C. M., Cavalcanti A. B., Oliveira R. P., Maccari J. G., Berto P. P, Martins L. A., Santos R. L. S., Ue L. Y., Hammes L. S., Sharshar T., Bozza F., Falavigna M. \& Teixeira C. (2018). Qualidade de vida pós-unidades de terapia intensiva: protocolo de estudo de corte multicêntrico para avaliação de desfechos em longo prazo em sobreviventes de internação em unidades de terapia intensiva brasileiras. Rev Bras Ter Intensiva, 30(4):405-413.

Roque, S. M., Souza, F. G. L., Taveira, G. S. (2017). Utilização do escore medical research council (MRC) e da dinamometria de preensão palmar no diagnóstico de fraqueza muscular adquirida em unidade de terapia intensiva (uti): Revisão Bibliográfica. Revista Inspirar. V. 10, n. 1, p. 1-14.

Sanabria A, Gómez X, Vega V, Dominguez LC, Osorio C. (2013). Prediction of prolonged mechanical ventilation for intensive care unit patients: A cohort study. Colomb Med. 44(3): 184-8

Santos L. J., Silveira F. S., Muller F. F., Araújo H. D., Comerlato J. B., Silva M. C. \& Silva P. B. (2017). Functional assessment of inpatients in the Intensive Care Unit of the University Hospital of Canoas. Rev. Fisioter Pesqui. 24(4): 437-443.

Souza F. S. H., Hojo N. S. S., Batista B. D. O., Silva C. M. \& Guidoni D. L. (2021). On the analysis of mortality risk factors for hospitalized COVID-19 patients: A data-driven study using the major Brazilian database. Plos One,16(3): 1-11.

Souza, M. O. C. (2021). Caracterização epidemiológica dos casos de covid-19 atendidos em um hospital de referência no Seridó potiguar. Monografia (Especialização) - Curso de Residência Multiprofissional em Atenção Básica, Universidade Federal do Rio Grande do Norte, Caicó.

Teich, V. D., Klajner, S., Almeida, F. A. S. D., Dantas, A. C. B., Laselva, C. R., Torritesi, M. G. \& Cendoroglo, M. (2020). Características epidemiológicas e clínicas dos pacientes com COVID-19 no Brasil. Einstein (São Paulo), 18.

Wang, D. et al. (2020). Clinical characteristics of 138 hospitalized patients with 2019 novel coronavirus-infected pneumonia in Wuhan, China. Jama, 323(11), 1061-1069. 